\title{
Diversity Gain Analysis of Distributed CDD Systems in Non-identical Frequency Selective Fading
}

\author{
Kyeong Jin Kim, Marco Di Renzo, Hongwu Liu, Philip V. Orlik, and H. Vincent Poor
}

\begin{abstract}
This paper investigates the diversity gain of a distributed cyclic delay diversity (CDD) scheme for cyclic-prefixed single carrier systems in non-identical frequency selective fading channels. Two conditions are used to obtain an equivalent channel matrix that is free of intersymbol interference. These conditions allows the system to achieve the maximum diversity order at a full rate in frequency selective fading channels. A given number of CDD transmitters is obtained from the set of cooperative transmitters in the system and is shown to be determined by the symbol block size and the maximum time dispersion of the channel. A new expression for the received signal-to-noise ratio (SNR) is derived by using order statistics. To estimate the achievable maximum diversity gain provided by the distributed CDD scheme, we employ asymptotic analysis in the high SNR regime. From the analytical framework, it is shown that the maximum diversity is achieved even for non-identical frequency selective fading channels. Link-level simulations are conducted to verify the maximum achievable diversity gain.
\end{abstract}

Index Terms-Distributed single carrier system, cyclic delay diversity, diversity gain, non-identical frequency selective fading.

\section{INTRODUCTION}

Several transmit diversity schemes such as distributed maximum ratio transmission (MRT) [1] and distributed spacetime-coding (STC) [2], [3] have been proposed. In contrast to the MRT scheme proposed by [4] and [5], distributed MRT can achieve the diversity gain for a general number of single antenna transmitters by increasing the receive signalto-noise ratio (SNR) over independent frequency selective fading channels. However, exact knowledge of channel state information (CSI) is required at the transmitters, which is a challenging problem in a distributed communications system. It is also known that a full rate orthogonal space-time block code (STBC) is not known for a general number of distributed transmitters.

As a solution of these problems, we consider cyclic delay diversity (CDD), which has been widely used in practical Orthogonal Frequency Division Multiplexing (OFDM)-based

K. J. Kim and P. V. Orlik are with Mitsubishi Electric Research Laboratories (MERL), Cambridge, MA, USA.

M. D. Renzo is with the Laboratoire des Signaux et Systèmes, CNRS, CentraleSupélec, Univ Paris Sud, Université Paris-Saclay, 3 rue Joliot Curie, Plateau du Moulon, 91192, Gif-sur-Yvette, France. (e-mail: marco.direnzo@12s.centralesupelec.fr).

H. Liu is with Shandong Jiaotong University, Jinan, China.

H. V. Poor is with the Department of Electrical Engineering, Princeton University, Princeton, NJ, USA.

This work was supported in part by the U.S. National Science Foundation under Grants CCF-1420575 and ECCS-1647198. wireless systems such as [6] and [7]. Although CDD requires lower complexity, in general, forward error correction (FEC) is also required for OFDM transmissions to convert spatial diversity into frequency diversity. Also, the conventional communications systems apply CDD between antennas that are installed at the same transmitter. In contrast to the employment of the conventional CDD, we investigate the distributed CDD (dCDD) that applies CDD between a single antenna transmitters with a reduced feedback overhead from the receiver.

Cyclic-prefixed single carrier (CP-SC) transmissions has been also proposed for several wireless systems [8] considering more practical issues such as peak-to-average ratio, power-backing off, and dynamic range of the linear amplifier [9]. For CP-SC transmissions, several works [10]-[13] have attempted to use CDD between antennas. Only a recent work [14] proposes a dCDD.

For frequency selective fading channels, it has been shown by [15] and [16] that the multipath diversity can be achieved without utilizing channel equalization [10]. Multiuser diversity is also exploited to achieve the maximum diversity by using either the best terminal selection [15] or best relay selection [16]. A similar transmit antenna selection (TAS) [17] is also proposed to achieve the diversity gain. However, these existing works assumes only identical frequency selective fading channels, and then derives the diversity gain. Although there are several works [18], [19] that derive the probability density function (PDF) of the partial sum of the order statistics, they mainly assume either identical Rayleigh fading or identical frequency selective fading channels, so that it is not straightforward to use them in diversity gain analysis. Thus, to the best our knowledge, the diversity gain analysis of the dCDD has never been investigated for non-identical frequency selective channels.

1) Motivated by the work in [12] and [13], we derive two conditions that make an equivalent channel matrix intersymbol interference (ISI) free, so that dCDD can achieve the maximum diversity promised by distributed CP-SC transmissions at a full rate. It also makes it possible to reduce feedback overhead from the receiver, and removes the use of FEC comparing with OFDM transmissions and other CP-SC transmissions schemes [11]. Thus, the $\mathrm{dCDD}$ can reduce the complexity of the transmitters to achieve the transmit diversity at a full rate.

2) We assume non-identical number of multipaths and non-identical frequency selective fading channels. Since dCDD maximizes the sum of the receive SNRs, it is 
necessary to use the order statistics for performance analysis. Comparing with the analysis [18], [19] that derives the PDF and cumulative distribution function (CDF) of the identical random variables, it is necessary to use the permanent matrix [20]. Although tedious manipulations are required, we can derive the closed-form expression for the PDF, and then CDF, which makes us to derive the outage probability. However, the derived expression is complex, in general, so that we also make an asymptotic outage probability analysis in the high SNR region. From this derivation, we are able to extract effectually the diversity gain achieved by dCDD in non-identical frequency selective fading channels. Link-level simulation results verify that the derived maximum diversity gain is correct.

Notation: $\boldsymbol{I}_{N}$ is an $N \times N$ identity matrix; $\mathbf{0}$ denotes an all zeros matrix of appropriate dimensions; $\mathcal{N}\left(\mu, \sigma^{2}\right)$ denotes the complex Gaussian distribution with the mean $\mu$ and the variance $\sigma^{2} ; \mathbb{C}^{m \times n}$ denotes the vector space of all $m \times n$ complex matrices; $F_{\varphi}(\cdot)$ denotes the $\mathrm{CDF}$ of the random variable $(\mathrm{RV}) \varphi$, whereas its PDF is denoted by $f_{\varphi}(\cdot)$; The binomial coefficient is denoted by $\left(\begin{array}{l}n \\ k\end{array}\right) \triangleq \frac{n !}{(n-k) ! k !}$.

\section{System AND CHANNEL MOdel}

A block diagram of the proposed cooperative CP-SC system is provided in Fig. 1. The CU provides perfect backhaul connections $\left\{b_{m}\right\}_{m=1}^{M}$ to $M$ single antenna transmitters $\left\{\mathrm{TX}_{m}\right\}_{m=1}^{M}$. We also assume one single antenna receiver, $\mathrm{R}$, in the considered system. As a channel, we assume independent and non-identically distributed (i.n.i.d.) frequency selective fading channels. They can be composed by different number of multipath components. A distance dependent path loss model is used for a large scale fading. Since transmitters are distributed in the system, different path losses can be assumed as well.

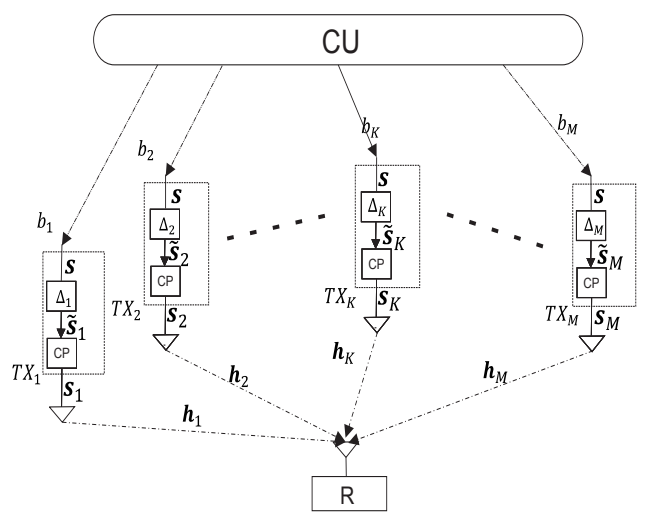

Fig. 1. Block diagram of the proposed dCDD-based cooperative single carrier system.

\section{A. Distributed CDD Operation}

Since we assume $M>K$ transmitters in the system, we need to select $K$ CDD transmitters that apply CDD processing. Using known pilot symbols, $\boldsymbol{p} \in \mathbb{C}^{Q \times 1}$, the receiver measures the receive SNR over the channel $\boldsymbol{h}_{k}$ connecting the $k$ th transmitter as

$$
\gamma_{k} \triangleq \frac{P_{T} \alpha_{k}\left\|\boldsymbol{h}_{k}\right\|^{2}}{\sigma_{z}^{2}}=\tilde{\alpha}_{k}\left\|\boldsymbol{h}_{k}\right\|^{2}
$$

where $\tilde{\alpha}_{k} \triangleq \frac{P_{T} \alpha_{k}}{\sigma_{z}^{2}}$ with $\alpha_{k}$ being used to model large scale fading as $\alpha_{k}=d_{k}^{-\epsilon}$ with the path loss exponent $\epsilon$ and the distance $d_{k}$ from the $k$ th CDD transmitter to the receiver. In addition, $P_{T}$ denotes the transmission power at the transmitters. An additive noise is modeled by $\boldsymbol{n}_{R} \sim \mathcal{N}\left(\mathbf{0}, \sigma_{z}^{2} \boldsymbol{I}_{Q}\right)$. We also assume that $E\{\boldsymbol{p}\}=\mathbf{0}, E\left\{\boldsymbol{p} \boldsymbol{p}^{H}\right\}=\boldsymbol{I}_{Q}$. The symbol block size will be denoted by $Q$ in the sequel. For a frequency selective fading channel $\boldsymbol{h}_{k}$, the CDF and PDF of $\gamma_{k}$ are, respectively, given by

$$
\begin{aligned}
F_{\gamma_{k}}(x) & =1-e^{-\frac{x}{\tilde{\alpha}_{k}}} \sum_{l=0}^{N_{k}-1} \frac{1}{l !}\left(\frac{x}{\tilde{\alpha}_{k}}\right)^{l} \text { and } \\
f_{\gamma_{k}}(x) & =\frac{x^{N_{k}-1}}{\Gamma\left(N_{k}\right)\left(\tilde{\alpha}_{k}\right)^{N_{k}}} e^{-\frac{x}{\bar{\alpha}_{k}}}
\end{aligned}
$$

where $\Gamma(\cdot)$ denotes the gamma function and $N_{k}$ denotes the number of multipath components of the channel $\boldsymbol{h}_{k}$.

Now for $M$ SNRs, we arranged them in ascending order of magnitude in such a way $0 \leq \gamma_{(1)} \leq \gamma_{(2)} \leq \cdots \leq \gamma_{(M)}$ with their corresponding indices as $\mathbb{X}_{I} \triangleq[(1),(2), \cdots,(M)]$. To reduce the feed back overhead, the receiver feeds back only $\mathbb{X}_{I}$ to the CU. Receiving $\mathbb{X}_{I}$, the CU will choose $K \leq M$ transmitters indexed by the last $K$ elements of $\mathbb{X}_{I}$.

To make the equivalent channel matrix free from ISI, we can find two conditions as follows:

$$
\begin{aligned}
& C_{1}: N_{p}=\max \left(N_{1}, \ldots, N_{M}\right) \\
& C_{2}: \Delta_{i}=(i-1) N_{p}
\end{aligned}
$$

where condition $C_{1}$ is required to remove ISI for CP-SC transmission [16] with the CP length $N_{p}$, whereas condition $C_{2}$ is required to form a non-overlapping equivalent channel vector in converting the multi-input single-output (MISO) channel into a single-input single-output (SISO) channel [13].

Based on conditions $C_{1}$ and $C_{2}$, the number of $K$ will be determined as follows:

$$
K=1+\left\lfloor\frac{Q}{N_{p}}\right\rfloor
$$

where $\lfloor\cdot\rfloor$ denotes the floor function. Since the CU needs to know $N_{p}$, the receiver needs to feedback this value to the CU as well. The receiver can determine this value via a channel sounding scheme. Having selected $K$ CDD transmitters, which are indexed by the last $K$ elements of $\mathbb{X}_{I}$, the CU forms a table for CDD delays, $\mathbb{X}_{\Delta} \triangleq\left\{\Delta_{1}, \ldots, \Delta_{K-1}, \Delta_{K}\right\}$, which will be used by $K$ CDD transmitters.

\section{B. Theoretical Background of the Distributed CDD}

The $k$ th CDD transmitter receives the transmission symbol block $s=\left[s_{1}, \ldots, s_{Q}\right]^{T} \in \mathbb{C}^{Q \times 1}$, and then applies CDD delay $\Delta_{k}$, which is expressed as follows:

$$
\tilde{\boldsymbol{s}}_{k}=\boldsymbol{P}_{Q}^{\Delta_{k}} \boldsymbol{s}
$$


where the permutation matrix, $\boldsymbol{P}_{Q}^{\Delta_{k}}$, is obtained by circularly shifting down the identity matrix $\boldsymbol{I}_{Q}$ by $\Delta_{k}$.

After removing the $\mathrm{CP}$ signal, the received signal from the $K$ CDD transmitters is given by

$$
\boldsymbol{r}=\sum_{k=1}^{K} \sqrt{P_{T} \alpha_{k}} \boldsymbol{H}_{k} \boldsymbol{P}_{Q}^{\Delta_{k}} \boldsymbol{s}+\boldsymbol{z}_{R}
$$

where $\boldsymbol{H}_{k}$ is the right circulant matrix determined by $\boldsymbol{h}_{k}$. The main objective of conditions $C_{1}$ and $C_{2}$ is to convert (7) into the following way

$$
\boldsymbol{r}=\boldsymbol{H}^{\mathrm{CDD}} \boldsymbol{s}+\boldsymbol{z}_{R}
$$

where $\boldsymbol{H}^{\mathrm{CDD}}$ is an equivalent right circulant channel matrix comprising frequency fading channels from $K$ CDD transmitters to the receiver. Its first column vector is specified as follows:

$$
\begin{aligned}
\boldsymbol{h}^{\mathrm{CDD} \triangleq} & {\left[\sqrt{P_{T} \alpha_{(1)}}\left(\boldsymbol{h}_{(1)}\right)^{T}, \mathbf{0}_{1 \times\left(N_{p}-N_{(1)}\right)},\right.} \\
& \sqrt{P_{T} \alpha_{(2)}}\left(\boldsymbol{h}_{(2)}\right)^{T}, \mathbf{0}_{1 \times\left(N_{p}-N_{(2)}\right)}, \ldots, \\
& \left.\sqrt{P_{T} \alpha_{(K)}}\left(\boldsymbol{h}_{(K)}\right)^{T}, \mathbf{0}_{1 \times\left(N_{p}-N_{(K)}\right)}\right]^{T} \in \mathbb{C}^{Q \times 1} .
\end{aligned}
$$

Thus, $\boldsymbol{H}^{\mathrm{CDD}}$ is exactly determined by $\boldsymbol{h}^{\mathrm{CDD}}$. Note that $\boldsymbol{h}^{\mathrm{CDD}}$ shows no ISI from CDD transmissions. To make the form of $\boldsymbol{h}^{\mathrm{CDD}}$ as in (9), $\boldsymbol{P}_{Q}^{\Delta_{k}}$ should be orthogonal and circulant.

For a general right circulant channel matrix, $\boldsymbol{H}_{c i r}$, when we apply the QR decomposition (QRD), we have

$$
\begin{aligned}
\boldsymbol{H}_{c i r} & =\boldsymbol{Q} \boldsymbol{R} \text { and } \\
\boldsymbol{H}_{c i r}^{\Delta_{k}} \triangleq \boldsymbol{H}_{c i r} \boldsymbol{P}_{Q}^{\Delta_{k}} & =\boldsymbol{Q}^{\Delta_{k}} \boldsymbol{R} .
\end{aligned}
$$

That is, the upper triangular matrix obtained from the QRD of the column permutated circulant matrix is independent of the column permutation. It has been verified that the receiver performance is mainly determined by the magnitude of the first diagonal element of the upper triangular matrix [21], as follows:

$$
\begin{aligned}
\operatorname{trace}\left(\left(\boldsymbol{H}_{c i r}^{\Delta_{k}}\right)^{H} \boldsymbol{H}_{c i r}^{\Delta_{k}}\right) & =\left(\boldsymbol{R}^{\Delta_{k}}(1,1)\right)^{2}=\sum_{l=1}^{N}\left|\boldsymbol{h}_{\text {cir }}^{\Delta_{k}}(l)\right|^{2} \\
& =(\boldsymbol{R}(1,1))^{2}=\sum_{l=1}^{N}\left|\boldsymbol{h}_{\text {cir }}(l)\right|^{2}(12)
\end{aligned}
$$

where $\boldsymbol{R}^{\Delta_{k}}(1,1)$ and $\boldsymbol{R}(1,1)$ are the first diagonal elements of matrices $\boldsymbol{R}^{\Delta_{k}}$ and $\boldsymbol{R}$, respectively. We can see that when we use the maximum likelihood detector in the receiver, a different deployment of the CDD delay does not change the receiver performance when the CDD delay satisfies conditions $C_{1}$ and $C_{2}$. That is, the CU has freedom in assigning a particular CDD delay $\Delta_{k}$ to a CDD transmitter.

\section{Performance Analysis in i.n.i.D. Channel}

To investigate the performance of the proposed dCDD, we need to know the distributions of the receive SNR.

\section{A. Receive SNR at the Receiver}

Based on (8), the receive SNR, aggregated by $K$ CDD transmitters, is given by [16]

$$
\begin{aligned}
S^{K} & =\sum_{k=1}^{K} \tilde{\alpha}_{(M-K+k)} \sum_{l=1}^{N_{(M-K+k)}}\left|\boldsymbol{h}_{(M-K+k)}(l)\right|^{2} \\
& =\sum_{k=1}^{K} \gamma_{(M-K+k)} \triangleq \sum_{k=1}^{K} \beta_{k}
\end{aligned}
$$

where $\beta_{k} \triangleq \gamma_{(M-K+k)}$ denotes the $k$ th largest SNR. Due to CDD operation, $\beta_{k}$ has a different distribution depending on its index $k$. For example, $\beta_{K}$ has the largest SNR; $\beta_{K-1}$ has the second largest SNR, and so on. Thus, $\sum_{k=1}^{K} \beta_{k}$ denotes the sum of the $K$ largest receive SNRs.

For $\beta_{1}<\beta_{2}<\ldots<\beta_{K}$, the joint PDF of $\beta_{1}, \beta_{2}, \ldots, \beta_{K}$ can be written as [20]:

$$
f_{\beta_{1}, \beta_{2}, \ldots, \beta_{K}}\left(x_{1}, x_{2}, \ldots, x_{K}\right)=\frac{1}{(M-K) !} \operatorname{Per} \boldsymbol{A}_{K}
$$

where

$$
\boldsymbol{A}_{K} \triangleq\left[\begin{array}{cccc}
F_{1}\left(x_{1}\right) & f_{1}\left(x_{1}\right) & \ldots & f_{1}\left(x_{K}\right) \\
F_{2}\left(x_{1}\right) & f_{2}\left(x_{1}\right) & \ldots & f_{2}\left(x_{K}\right) \\
\vdots & \vdots & \ldots & \vdots \\
F_{M}\left(x_{1}\right) & f_{M}\left(x_{1}\right) & \ldots & f_{M}\left(x_{K}\right) \\
\underbrace{C-K} & \underbrace{1} & \underbrace{1}
\end{array}\right]
$$

with $F_{j}(\cdot)$ and $f_{j}(\cdot)$ are respectively denoting the $\mathrm{CDF}$ and PDF of $\gamma_{j}$, the receive SNR before applying dCDD operation. Their expressions are provided in (2). Also, we define $\left[\begin{array}{cc}a_{11} & a_{12} \\ \vdots & \vdots \\ a_{C 1} & a_{C 2} \\ \underbrace{i} & \underbrace{j}\end{array}\right]$ denoting $i$ copies of the first column vector $\left[a_{11}, a_{21}, \ldots, a_{C 1}\right]^{T}$ and $j$ copies of the second column vector $\left[a_{12}, a_{22}, \ldots, a_{C 2}\right]^{T}$, and so on. The permanent of the square matrix $\boldsymbol{A}$, denoted by $\operatorname{Per} \boldsymbol{A}$, is defined similar to the definition of the matrix determinant except that all signs are taken to be positive [20]. For example, for a square matrix $\boldsymbol{A}$, given by $\boldsymbol{A}=\left[\begin{array}{cc}a & b \\ c & d \\ 1 & \underbrace{1}\end{array}\right]$, we have $\operatorname{Per} \boldsymbol{A}=a d+b c$. With some manipulations, a desired compact expression for $\operatorname{Per} \tilde{\boldsymbol{A}}_{K} \triangleq \frac{\operatorname{Per} \boldsymbol{A}_{K}}{(M-K) !}$ can be given by (16) at the next page. For ease of analysis and expression, we assume $N_{j}=N_{h} \forall j$, and introduce the notation $\mathbb{X}_{M} \triangleq\{1, \ldots, M\}$ and $\mathbb{X}_{p} \triangleq \mathbb{X}_{M}-$ $\left\{i_{1}, \ldots, i_{M-K}\right\}$. Also, the list of all possible permutations of the elements of $\mathbb{X}_{p}$ is denoted by $\mathbb{P}_{p} \triangleq \operatorname{Perms}\left(\mathbb{X}_{p}\right)$, where $q$ denotes the $q$ th permutation of $\mathbb{P}_{p}$. In addition, $k_{l, q}$ denotes the $l$ th element of permutation $q$. Having applied (16), the moment 


$$
\begin{aligned}
\operatorname{Per} \tilde{\boldsymbol{A}}_{K}= & \sum_{\substack{i_{1}, i_{2}, \ldots, i_{M-K} \\
1 \leq i_{1}<i_{2}<\ldots<i_{M-K} \leq M}} \sum_{q \in \mathbb{P}_{p}} \prod_{j=1}^{M-K} F_{i_{j}}\left(x_{1}\right) f_{k_{1, q}}\left(x_{1}\right) \prod_{l=2}^{K} f_{k_{l, q}}\left(x_{l}\right) \\
= & \sum_{\substack{i_{1}, i_{2}, \ldots, i_{M-K} \\
1 \leq i_{1}<i_{2}<\ldots<i_{M-K} \leq M}} \sum_{q \in \mathbb{P}_{p}} \prod_{j=1}^{M-K}\left(1-e^{-\frac{x_{1}}{\bar{\alpha}_{i_{j}}}} \sum_{l=0}^{N_{i_{j}}-1} \frac{\left(x_{1}\right)^{l} \tilde{\alpha}_{i_{j}}^{-l}}{\Gamma(l+1)} \frac{\left(x_{1}\right)^{N_{k_{1, q}}-1} e^{-\frac{x_{1}}{\alpha_{k_{1, q}}}}}{\Gamma\left(N_{k_{1, q}}\right)\left(\tilde{\alpha}_{k_{1, q}}\right)^{N_{k_{1, q}}}} \prod_{l=2}^{K} \frac{\left(x_{l}\right)^{N_{k_{l, q}}-1} e^{-\frac{x_{l}}{\alpha_{k_{l, q}}}}}{\Gamma\left(N_{k_{l, q}}\right)\left(\tilde{\alpha}_{k_{l, q}}\right)^{N_{k_{l, q}}}} \cdot(16)\right.
\end{aligned}
$$

generating function (MGF) of the RV $S_{K}$ can be evaluated as follows:

$$
\begin{aligned}
\Phi_{S^{K}}(s)= & \int_{0}^{\infty} \int_{0}^{x_{K}} \ldots \int_{0}^{x_{3}} \int_{0}^{x_{2}} e^{-s\left(x_{1}+\ldots+x_{K}\right)} \\
& \operatorname{Per} \tilde{\boldsymbol{A}}_{K} d x_{1} d x_{2} \ldots d x_{K-1} d x_{K} .
\end{aligned}
$$

Thus, the closed-form requires $K$-fold nested integrals. One example for two CDD transmitters is provided in the following theorem.

Theorem 1: The CDF of the receive SNR achievable by two CDD transmitters in the i.n.i.d. frequency selective fading channel is given by (18) at the next page. In (18), we have defined $D_{1} \triangleq \frac{q_{1}}{\tilde{\alpha}_{i_{1}}}+\ldots+\frac{q_{M-K}}{\tilde{\alpha}_{i_{M-K}}}+\frac{1}{\tilde{\alpha}_{k_{1, q}}}, \tilde{m}_{1} \triangleq \tilde{q}_{1}+\ldots+$ $\tilde{q}_{M-K}+N_{h}, \tilde{q}_{l} \triangleq \sum_{t_{l}=0}^{N_{h}-1} t_{l} q_{l, t_{l}+1}$, and $D_{2} \triangleq \frac{1}{\tilde{\alpha}_{k_{2, q}}}$. In addition, $\gamma_{l}(\cdot, \cdot)$ denotes the lower incomplete gamma function.

Proof: See [14].

\section{Asymptotic Diversity Gain AnALYsis}

Since the outage probability is a special case of the CDF, we can readily derive its closed-form expression. For a given outage threshold, $\gamma_{\mathrm{th}}$, the outage probability is given by $O_{\text {out }}\left(\gamma_{\text {th }}\right)=F_{S^{K}}\left(\gamma_{\mathrm{th}}\right)$. Although we can derive the closedform expression for the outage probability, it is not easy to extract the diversity gain. Thus, we conduct an asymptotic outage probability analysis in the high SNR region.

In the high SNR region, we can see that $O_{\text {out }}\left(\gamma_{\text {th }}\right)$ is the sum of the different combinations of $\left(x_{1}, \ldots, x_{K}\right)$ without changing the diversity gain. Thus, we mainly focus on the major diagonal of $\boldsymbol{A}_{k}$, namely $\Delta_{d} \triangleq \prod_{j=1}^{M-K} F_{j}\left(x_{1}\right) f_{M-K+j}\left(x_{1}\right) \prod_{l=2}^{K} f_{M-K+l}\left(x_{l}\right)$. Based on this knowledge, we can derive the diversity gain of dCDD in the non-identical frequency selective fading channel.

Theorem 2: The achievable diversity gain by the proposed $\mathrm{dCDD}$ in the high SNR region and in non-identical frequency selective fading channel is given by $G_{d}=\sum_{j=1}^{M} N_{j}$.

Proof: See Appendix A.

Theorem 2 indicates that the number of transmitters in the system and the number of multipath element of each channel are two key parameters that determine the maximum diversity gain of the CP-SC system with dCDD. We can see that the number of multipath element of a channel give conflicting effects on the maximum diversity gain. As the multipath element increases, a larger multipath diversity gain can be obtained. However, the number of CDD transmitters is decreased, so that the transmit diversity gain is decreased.

\section{Simulation Results}

In the following link-level simulations, we employ Quadrature Phase Shift Keying (QPSK) to the data symbols. The curves obtained by the link-level simulations are denoted by Ex. Analytically performance curves denoted by An. Asymptotically derived curves are denoted by As. The transmission block size for CP-SC transmission is $Q=64$. The transmission power is assumed to be $P_{T}=1$ for all transmitters. In addition, a SNR threshold causing outage is fixed at $1 \mathrm{~dB}$. For Figs. 2 and 3 , we assume $\alpha_{k}=\{0.12,0.13,0.14,0.15\}$ with the same $N_{h}=N_{j}, \forall j$.

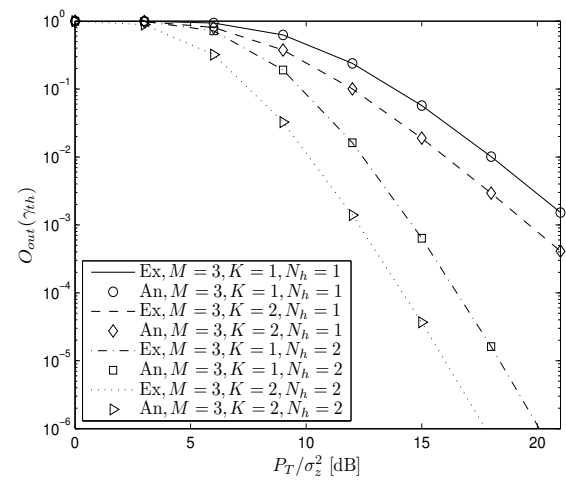

Fig. 2. Outage probability for various cases.

Fig. 2 shows the correctness of the derived outage probability comparing with the exact outage probability. This figure also shows that as the more CDD transmitters are involved in dCDD operation, $K$, at the same number of transmitters, $M$, a lower outage probability is obtained. We can also see that for the same number of CDD transmitters, a more number of transmitters results in a lower outage probability.

Fig. 3 shows the diversity gain analysis of the CP-SC system based on the asymptotic outage probability. Comparing with the asymptotic analysis, we can see the diversity gain $G_{d}$, which was verified by Theorem 2 . For $\left\{\left(M, K, N_{h}\right) \mid(3,1,1),(3,2,1),(3,2,2)\right\}$, asymptotic diversity gain can be measured as $G_{d}=\{2.8065,2.8864,5.8574\}$, so that $G_{d}=M N_{h}$ can be verified.

In Fig. 4, we use a different values of $\alpha_{k}$ as $\alpha_{k}=\{0.2617,0.3728,0.2574,0.2450\}$ and non-identical number of multipath elements as $N_{j}=\{2,3,1,2\}$. For $\{(M, K) \mid(2,1),(2,2),(3,1)\}$, we can measure respectively $G_{d}$ in the high SNR as: $G_{d}=\{4.65,4.11,5.3857\}$. Thus, we can verify that $G_{d}=\sum_{j=1}^{M} N_{j}$. From Figs. 3 and 4 , the 


$$
\begin{aligned}
& F_{S^{K=2}}(x)=\sum_{\substack{i_{1}, i_{2}, \ldots, i_{M-2} \\
1 \leq i_{1}<i_{2}<\ldots<i_{M-2} \leq M}} \sum_{q \in \mathbb{P}_{p}} \sum_{q_{1}=0}^{1} \ldots \sum_{q_{M-2}=0}^{1}\left(\begin{array}{c}
1 \\
q_{1}
\end{array}\right) \ldots\left(\begin{array}{c}
1 \\
q_{M-2}
\end{array}\right)(-1)^{q_{1}+\ldots+q_{M-2}} \\
& \sum_{\substack{q_{1,1}, \ldots, q_{1, N_{h}} \\
q_{1,1}+\ldots+q_{1, N_{h}=q_{1}}}} \ldots \sum_{\substack{q_{M-2,1}, \ldots, q_{M-2, N_{h}} \\
q_{M-2,1}+\ldots+q_{M-2, N_{h}}=q_{M-2}}} \prod_{j=1}^{M-K}\left(\frac{q_{j} !}{q_{j, 1} ! \ldots q_{j, N_{h}} !}\right) \\
& \prod_{j=1}^{M-2} \prod_{t_{j}=0}^{N_{h}-1}\left(\frac{1}{t_{j} !}\right)^{q_{j, t_{j}+1}} \prod_{j=1}^{M-2}\left(\frac{1}{\tilde{\alpha}_{i, j}}\right)^{\tilde{q}_{j}}\left(\frac{1}{\tilde{\alpha}_{k_{1, q}}}\right)^{N_{h}} \frac{\Gamma\left(\tilde{m}_{1}\right)}{\Gamma\left(N_{h}\right)}\left(\frac{1}{\tilde{\alpha}_{k_{2, q}}}\right)^{N_{h}} \\
& {\left[\sum_{f=1}^{\tilde{m}_{1}}(-1)^{\tilde{m}_{1}-f}\left(D_{2}-D_{1}\right)^{-\left(\tilde{m}_{1}+N_{h}-f\right)}\left(\begin{array}{c}
\tilde{m}_{1}+N_{h}-f-1 \\
\tilde{m}_{1}-f
\end{array}\right) \frac{\gamma_{l}\left(f, D_{1} x\right)}{\Gamma(f)\left(D_{1}\right)^{f}}+\right.} \\
& \sum_{f=1}^{N_{h}}(-1)^{N_{h}-f}\left(D_{1}-D_{2}\right)^{-\left(\tilde{m}_{1}+N_{h}-f\right)}\left(\begin{array}{c}
\tilde{m}_{1}+N_{h}-f-1 \\
N_{h}-f
\end{array}\right) \frac{\gamma_{l}\left(f, D_{2} x\right)}{\Gamma(f)\left(D_{2}\right)^{f}}-\sum_{b=0}^{\tilde{m}_{1}-1} \frac{(2)^{-b-N_{h}} \Gamma\left(b+N_{h}\right)}{\Gamma(b+1) \Gamma\left(N_{h}\right)} \\
& {\left[\sum_{f=1}^{\tilde{m}_{1}-b}(-1)^{\tilde{m}_{1}-b-f}\left(D_{2} / 2-D_{1} / 2\right)^{-\left(\tilde{m}_{1}+N_{h}-f\right)}\left(\begin{array}{c}
\tilde{m}_{1}+N_{h}-f-1 \\
\tilde{m}_{1}-b-f
\end{array}\right) \frac{\gamma_{l}\left(f, D_{1} x\right)}{\Gamma(f)\left(D_{1}\right)^{f}}+\right.} \\
& \left.\left.\sum_{f=1}^{N_{h}+b}(-1)^{N_{h}+b-f}\left(D_{1} / 2-D_{2} / 2\right)^{-\left(\tilde{m}_{1}+N_{h}-f\right)}\left(\begin{array}{c}
\tilde{m}_{1}+N_{h}-f-1 \\
N_{h}+b-f
\end{array}\right) \frac{\gamma_{l}\left(f,\left(D_{1} / 2+D_{2} / 2\right) x\right)}{\Gamma(f)\left(\left(D_{1} / 2+D_{2} / 2\right)\right)^{f}}\right]\right] .
\end{aligned}
$$

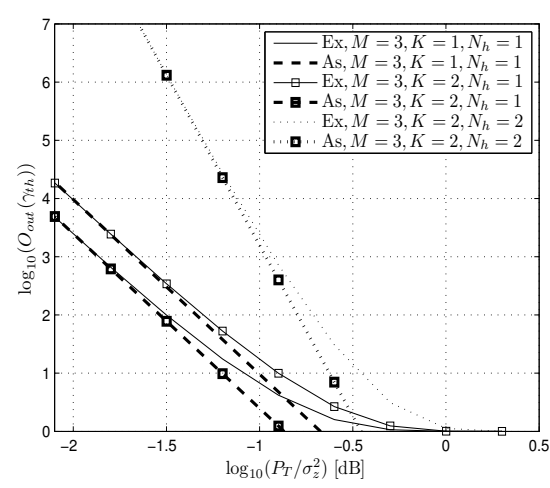

Fig. 3. Diversity gain analysis for various cases.

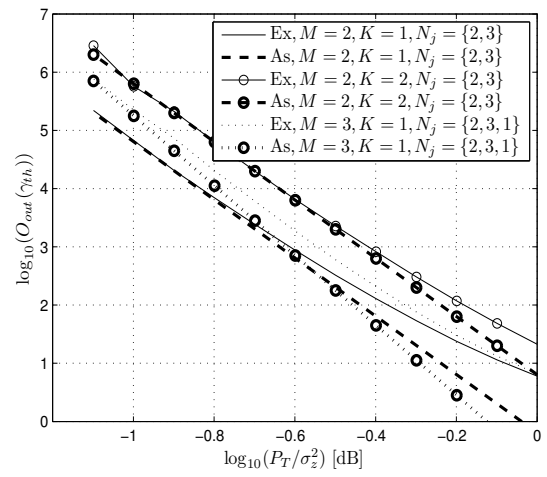

Fig. 4. Diversity gain analysis for various cases.

large scale fading does not influence on the diversity gain of the proposed system. If we compare the diversity gain with those of [15] and [16], dCDD provides the same maximum diversity gain. However, since dCDD can use more than one transmitters for CDD operation, a larger coding gain can be achieved. This can be easily observable from Figs. 3 and 4. That is, dCCC can achieve the maximum diversity gain and the coding gain simultaneously in the non-identical frequency selective fading channels.

\section{CONCLUSIONS}

In this paper, we have derived the diversity gain of dCDD in the non-identical frequency selective fading channels. It has been shown that dCDD makes it possible for the cooperative $\mathrm{CP}-\mathrm{SC}$ system to achieve the maximum diversity gain and coding gain. This is possible since dCDD converts the MISO channel into an ISI-free SISO channel over the frequency selective fading channel. Thus, a lower outage probability can be achieved over the cooperative diversity schemes that achieve only the maximum diversity gain. The diversity gain has been derived and verified via link level simulations.

\section{APPENDIX A: DERIVATION OF THEOREM 2}

In the high SNR region, we approximate $F_{j}\left(x_{1}\right)$ as [15]

$$
F_{j}\left(x_{1}\right) \stackrel{x_{1}}{\rightarrow} \approx 0 \frac{\left(x_{1} / \tilde{\alpha}_{j}\right)^{N_{j}}}{\left(N_{j}+1\right)} .
$$

Thus, $\Delta_{d}$ is approximated as

$$
\begin{aligned}
& \Delta_{d} \approx \prod_{l=1}^{M-K}\left(\frac{1}{N_{l}+1}\right)\left(\frac{1}{\tilde{\alpha}_{l}}\right)^{N_{l}} x_{1}^{\sum_{j=1}^{M-K} N_{j}} \\
& \frac{e^{-\frac{x_{1}}{\tilde{\alpha}_{M-K+1}}} x_{1}^{M-K+1}}{\Gamma(M-K+1)\left(\tilde{\alpha}_{M-K+1}\right)^{N_{M-K+1}}} \prod_{l=2}^{K} f_{M-K+l}\left(x_{l}\right) .
\end{aligned}
$$


Its first approximate contribution to the MGF is computed as

$$
\begin{aligned}
& \Phi_{S^{K}}(s) \propto \int_{0}^{\infty} f_{M}\left(x_{K}\right) e^{-s x_{K}} d x_{K} \ldots \prod_{l=1}^{M-K}\left(\frac{1}{N_{l}+1}\right) \\
& \prod_{l=1}^{M-K}\left(\frac{1}{\tilde{\alpha}_{l}}\right)^{N_{l}} \frac{\prod_{l=2}^{K} f_{M-K+l}\left(x_{l}\right)}{\Gamma(M-K+1)\left(\tilde{\alpha}_{M-K+1}\right)^{N_{M-K+1}}} \\
& \int_{0}^{x_{2}} e^{-x_{1}\left(s+1 / \tilde{\alpha}_{M-K+1}\right)} x_{1}^{\sum_{j=1}^{M-K+1} N_{j}-1} d x_{1} \\
\propto & \int_{0}^{\infty} f_{M}\left(x_{K}\right) e^{-s x_{K}} d x_{K} \cdots \prod_{l=1}^{M-K}\left(\frac{1}{N_{l}+1}\right) \\
& \prod_{l=1}^{M-K+1}\left(\frac{1}{\tilde{\alpha}_{l}}\right)^{N_{l}} \frac{\prod_{l=2}^{K} f_{M-K+l}\left(x_{l}\right)}{\Gamma(M-K+1)\left(\sum_{j=1}^{M-K+1} N_{j}+1\right)} .
\end{aligned}
$$

Keep continuing the computation of (A.3), we can have the following

$$
\begin{aligned}
& \Phi_{S^{K}}(s) \stackrel{\propto}{\sim} \prod_{l=1}^{M-K}\left(\frac{1}{N_{l}+1}\right) \prod_{l=1}^{K}\left(\frac{1}{\Gamma(M-K+l)}\right) \\
& \prod_{l=1}^{K-1}\left(\frac{1}{\sum_{j=1}^{M-K+l} N_{j}+1}\right) \prod_{l=1}^{M}\left(\frac{1}{\tilde{\alpha}_{l}}\right)^{N_{l}} \Gamma\left(\sum_{j=1}^{M} N_{j}\right) \\
& \left(s+\frac{1}{\tilde{\alpha}_{M}}\right)^{-\sum_{j=1}^{M} N_{j}} .
\end{aligned}
$$

Based on (A.4), the CDF can be derived as follows:

$$
\begin{aligned}
F_{S^{K}}(x) \stackrel{\propto}{\sim} & \prod_{l=1}^{M-K}\left(\frac{1}{N_{l}+1}\right) \prod_{l=1}^{K}\left(\frac{1}{\Gamma(M-K+l)}\right) \\
& \prod_{l=1}^{K-1}\left(\frac{1}{\sum_{j=1}^{M-K+l} N_{j}+1}\right) \prod_{l=1}^{M}\left(\frac{1}{\tilde{\alpha}_{l}}\right)^{N_{l}} \\
& \left(\frac{1}{\tilde{\alpha}_{M}}\right)^{-\sum_{j=1}^{M} N_{j}} \gamma_{l}\left(\sum_{j=1}^{M} N_{j}, \frac{x}{\tilde{\alpha}_{M}}\right),
\end{aligned}
$$

which is further approximated as

$$
\begin{aligned}
& F_{S^{K}}(x) \stackrel{\propto}{\sim} \prod_{l=1}^{M-K}\left(\frac{1}{N_{l}+1}\right) \prod_{l=1}^{K}\left(\frac{1}{\Gamma(M-K+l)}\right) \\
& \prod_{l=1}^{K}\left(\frac{1}{\sum_{j=1}^{M-K+l} N_{j}+1}\right) \prod_{l=1}^{M}\left(\frac{1}{\tilde{\alpha}_{l}}\right)^{N_{l}} x^{\sum_{l=1}^{M} N_{l}} .
\end{aligned}
$$

Thus, at a given value of $x=r_{\mathrm{th}}$, (A.6) is evaluated as follows:

$$
\begin{aligned}
& F_{S^{K}}\left(r_{\mathrm{th}}\right) \stackrel{\propto}{\sim} \prod_{l=1}^{M-K}\left(\frac{1}{N_{l}+1}\right) \prod_{l=1}^{K}\left(\frac{1}{\Gamma(M-K+l)}\right) \\
& \prod_{l=1}^{K}\left(\frac{1}{\sum_{j=1}^{M-K+l} N_{j}+1}\right) \prod_{l=1}^{K}\left(\frac{1}{\tilde{\alpha}_{l}}\right)^{N_{l}} r_{\mathrm{th}}^{\sum_{l=1}^{M} N_{l}} .
\end{aligned}
$$

Converting $F_{S^{K}}\left(r_{\mathrm{th}}\right)$ in the $\log -\log$ scale, we can have

$$
\log \left(F_{S^{K}}\left(r_{\mathrm{th}}\right)\right) \stackrel{\sim}{\sim} C_{1}-\sum_{l=1}^{M} N_{l} \log \left(\frac{P_{T}}{\sigma_{z}^{2}}\right)
$$

where $C_{1}=-\sum_{l=1}^{M-K} \log \left(\left(N_{j}+1\right)\right)-$ $\sum_{l=1}^{K} \log \left(\Gamma\left(N_{M-K+l}\right)\right)-\sum_{l=1}^{K} \log \left(\sum_{j=1}^{M-K+l} N_{j}+1\right)-$ $\sum_{j=1}^{M} N_{j} \log \left(\alpha_{k}\right)+\log \left(\sum_{j=1}^{M} N_{j} \gamma_{\mathrm{th}}\right)$. Thus, we can extract diversity gain $G_{d}$.

\section{REFERENCES}

[1] K. J. Kim, T. Khan, and P. Orlik, "Performance analysis of cooperative systems with unreliable backhauls and selection combining," IEEE Trans. Veh. Technol., vol. 66, no. 3, pp. 2448-2461, Mar. 2017.

[2] J. N. Laneman and G. W. Wornell, "Energy-efficient antenna sharing and relaying for wireless networks," in Proc. IEEE Wireless Communications and Networking Conf., Chicago, IL, Oct. 2000, pp. 7-12.

[3] M. O. Astal and A. M. Abu-Hudrouss, "SIC detector for 4 relay distributed space-time block coding under quasi-synchronization," IEEE Commun. Lett., vol. 15, no. 10, pp. 1056-1058, Oct. 2011.

[4] J. K. Cavers, "Single-user and multiuser adaptive maximal ratio transmission for Rayleigh channels," IEEE Trans. Veh. Technol., vol. 49, no. 6, pp. 2043-2050, Nov. 2000.

[5] T. K. Y. Lo, "Maximum ratio transmission," IEEE Trans. Commun., vol. 47, no. 10, pp. 1458-1461, Oct. 1999.

[6] IEEE, "Wireless LAN medium access control (MAC) and physical layer (PHY) specifications-Amendment 4: Enhancements for very high throughput for operation in bands below $6 \mathrm{GHz}$," IEEE Standard 802.11ac-2013, Part 11, 2009.

[7] — , "Wireless LAN medium access control (MAC) and physical layer (PHY) specifications - Amendment 5: Enhancements for higher throughput," IEEE Standard 802.11n-2009, Part 11, 2009.

[8] IEEE P802.11ad/D0.1, "Wireless LAN medium access control (MAC) and physical layer (PHY) specifications: Enhancements for very high throughput in the $60 \mathrm{GHz}$ band," Jun. 2010.

[9] S. Kato, H. Harada, R. Funada, T. Baykas, C. S. Sum, J. Wang, and M. A. Rahman, "Single carrier transmission for multi-gigabit $60-\mathrm{GHz}$ WPAN systems," IEEE J. Sel. Areas Commun., vol. 27, no. 8, pp. 14661478, Oct. 2009.

[10] Y.-C. Liang, W. S. Leon, Y. Zeng, and C. Xu, "Design of cyclic delay deievsity for single carrier cyclic prefix (sccp) transmissions with blockiterative GDFE (BI-GDFE) receiver," IEEE Trans. Wireless Commun., vol. 7, no. 2, pp. 677-684, Feb. 2008.

[11] A. H. Mehana and A. Nosratinia, "Single-carrier frequency-domain equalizer with multi-antenna transmit diversity," IEEE Trans. Wireless Commun., vol. 12, pp. 388-397, Jan. 2013.

[12] Q. Li, Q. Yan, K. C. Keh, K. H. Li, and Y. Hu, "A multi-relay-selection scheme with cyclic delay diversity," IEEE Commun. Lett., vol. 17, no. 2, pp. 349-352, Feb. 2013.

[13] U.-K. Kwon and G.-H. Im, "Cyclic delay diversity with frequency domain Turbo equalization for uplink fast fading channels," IEEE Commun. Lett., vol. 13, no. 3, pp. 184-186, Mar. 2009.

[14] K. J. Kim, M. D. Renzo, H. Liu, P. V. Orlik, and H. V. Poor, "Performance analysis of distributed single carrier systems with distributed cyclic delay diversity," IEEE Trans. Commun., 2017, under publication.

[15] K. J. Kim, T. A. Tsiftsis, and H. V. Poor, "Power allocation in cyclic prefixed single-carrier relaying systems," IEEE Trans. Wireless Commun., vol. 10, no. 7, pp. 2297-2305, Jul. 2011.

[16] K. J. Kim and T. A. Tsiftsis, "On the performance of cyclic prefix-based single-carrier cooperative diversity systems with best relay selection," IEEE Trans. Wireless Commun., vol. 10, no. 4, pp. 1269-1279, Apr. 2011.

[17] P. L. Yeoh, M. Elkashlan, K. J. Kim, T. Q. Duong, and G. K. Karagiannidis, "Transmit antenna selection in cognitive MIMO relaying with multiple primary transceivers," IEEE Trans. Veh. Technol., vol. 65, no. 1 , pp. 483-489, 2016

[18] K. Alam and K. T. Wallenius, "Distribution of a sum of order statistics," Scandinavian Journal of Statistics, vol. 6, no. 3, pp. 845-855, 1979.

[19] E. Bedeer and M. J. Hossain, "Performance of low-complexity uniform power loading OFDM systems with reduced feedback over Rayleigh fading channels," IEEE Trans. Wireless Commun., vol. 15, no. 6, pp. 3783-3795, Jun. 2016.

[20] N. Balakrishnan, "Permanents, order statistics, outliers, and robustness," Rev. Mat. Complu., vol. 20, pp. 7-107, 2007.

[21] K. J. Kim and T. A. Tsiftsis, "Performance analysis of QRD-based cyclically prefixed single-carrier transmissions with opportunistic scheduling," IEEE Trans. Veh. Technol., vol. 60, pp. 328-333, Jan. 2011. 